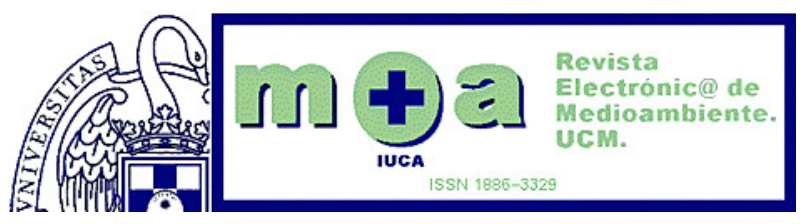

\title{
MODELOS DE ACTUACIÓN CONTRADICTORIOS: DIEZ AÑOS DESPUÉS DEL PRESTIGE
}

\author{
J. A. SOTELO NAVALPOTRO \\ Instituto de Ciencias Ambientales (UCM) \\ Grupo de Investigación de la UCM: "Desarrollo y Gestión Ambiental del Territorio" \\ jasotelo@ghis.ucm.es
}

Recibido: 28-05-2012

Aceptado: 15-06-2012

\section{RESUMEN}

En el presente artículo se estudia, una realidad marcada por unos modelos de desarrollo en los que uno de los aspectos más característico es el de un creciente y, en ocasiones, desmesurado y no siempre controlado desarrollo tecnológico, de cuyos efectos hemos empezado a tomar conciencia. Una de las causas ha sido la consideración de las anomalías y variantes que, como consecuencia del referido fenómeno, se están produciendo en nuestro entorno; todo ello resumido en la creciente preocupación por el medio ambiente, en el contexto de la Geografía Regional. Un buen ejemplo lo encontramos en las consecuencias generadas por el hundimiento del Prestige hace diez años. Del estudio de esta tragedia se colige que se debería plantear y generar importantes cambios en la política energética, pues resulta un despropósito que tras la catástrofe del Prestige, se haya mantenido una política que favorezca, cuando menos, que España cumpla los compromisos asumidos del Protocolo de Kioto, ahora que nos encontramos ante Río+20.

Palabras clave: Modelos de Desarrollo, gobernanza, crisis ambiental, convenciones internacionales, Prestige.

\section{Conflicting performance models: ten years after the Prestige}

\section{ABSTRACT}

This article discusses, a reality marked by patterns of development in which one of the most characteristic is that of a growing and, sometimes, exaggerated and not always controlled technological development, whose effects have begun to realize. One reason has been the consideration of anomalies and variants, as a consequence of that phenomenon, occurring in our environment, all summarized in the growing 
concern for the environment in the context of regional geography. A good example is in the consequences created by the sinking of the Prestige, last ten years. The study of this tragedy, it follows that should be raised and generate significant changes in energy policy, because it is an absurdity that after the Prestige disaster, has maintained a policy that favors at least that Spain fulfills the commitments of the Protocol Kyoto, now that we are facing Rio +20 .

Keywords: Development Models, governance, environmental crisis, international conventions, Prestige.

\section{PRIMERA APROXIMACIÓN}

A la hora de reflexionar sobre los denominados "Riesgos Naturales y Tecnológicos" que afectan o pueden incidir en nuestro país, a pesar de todos los pesares debemos de ser optimistas. No podemos permitirnos el lujo de afirmar con Chesterton (Manalive) "sí, somos tan vulgares y violentos, hemos hecho tantas cosas malas, que resulta vergonzoso que tengamos razón". Hoy el acceso a la información ambiental es ya un derecho, y como tal debemos transmitírselo a nuestros alumnos y alumnas, aplicando tanto la "Ley sobre derecho de acceso a la información en materia de medio ambiente" como el "Convenio de Aarhus".

Si tomamos como punto de partida una publicación reciente del Ministerio de Medio Ambiente (AA. VV. (2011). Perfil ambiental de España. Informe basado en Indicadores), observaremos que los temporales marítimos y las inundaciones son la causa principal de la pérdida de vidas humanas por riesgos naturales

Como consecuencia del conjunto de fenómenos naturales considerados, en España se han producido 928 fallecimientos en el período 19952011 , en concreto, en el período considerado las inundaciones han provocado más del $39 \%$ de las víctimas, los temporales marítimos más del $25 \%$ y las tormentas (rayos y vientos fuertes) cerca del $28 \%$. Un caso especial en el conjunto de los riesgos naturales es el que presentan las avenidas y las inundaciones, especialmente en el área mediterránea. Las características meteorológicas en estas zonas dan lugar a que se produzcan en pocas horas precipitaciones que alcanzan valores superiores al promedio anual. Estas lluvias extraordinarias provocan caudales extremos habitualmente denominados crecidas, avenidas o riadas que al desbordar los cauces habituales provocan la inundación de los terrenos adyacentes. En muchas ocasiones éstos han sido invadidos antes por edificaciones e instalaciones, con el consiguiente aumento de riesgos.

En nuestro país, las zonas de mayor desproporción entre los caudales ordinarios y los extraordinarios, y, por tanto, zonas de riesgo con alta probabilidad de sufrir avenidas, son la cuenca del Ebro, una franja variable 
del litoral Mediterráneo (incluidas las islas Baleares), zonas localizadas de las islas Canarias y otros puntos dispersos por la Península.

En la mayoría de los casos las consecuencias de las avenidas se valoran por los daños materiales que provocan, aunque no siempre es así. El caso más dramático de los últimos años fue la catástrofe de Biescas (Barranco de Arás, Huesca, en agosto de 1996) en la que se produjeron 87 víctimas mortales. Otro ejemplo destacable se produjo en marzo de 2002, cuando una gran tromba de agua sobre Santa Cruz de Tenerife originó 8 muertos, 30 heridos y daños materiales que superaron los 100 millones de euros.

En el período 1990-2003, el número total de víctimas mortales por avenidas e inundaciones en España ha sido de 256, destacando el elevado número de Aragón (por la ya mencionada catástrofe de Biescas), Andalucía, Cataluña y Extremadura, todas ellas con más de veinte víctimas mortales.

En primer lugar, nos encontramos con la Gota Fría. Nos referimos al fenómeno que se caracteriza por producir precipitaciones intensas de poca duración (fuertes tormentas) que afectan a una zona poco extensa. Generalmente, se producen en la costa mediterránea al final del verano e inicio del otoño (meses de septiembre y octubre principalmente), aunque puede desarrollarse también en otros lugares. Por su frecuencia, periodicidad y consecuencias salta a los medios de comunicación y atrae la atención de la opinión pública. En muchas ocasiones las gotas frías dan lugar a grandes desastres, como la enorme crecida del río Júcar que rompió la presa de Tous en octubre de 1982, las grandes riadas de Bilbao y otras zonas del País Vasco (que en agosto de 1983 originaron más de 30 muertos) o las que inundaron ciudades como Valencia, Alicante, Almería o Tarrasa.

La gota fría se origina cuando el aire cargado con la humedad del mar se introduce en el interior del continente, donde choca con el relieve o con masas de aire más frías. Esto provoca un ascenso del mismo y una rápida condensación, originando lluvias muy intensas. En el ámbito científico existen ciertas dudas sobre la utilización del término "gota fría", ya que no siempre se adecua a su concepción y origen teórico.

Igualmente, es importante, en segundo lugar, referirnos a los "períodos de sequía". El indicador establece si un año hidrológico puede ser considerado como un año de sequía generalizada en la España peninsular. Para su cálculo se han utilizado las medias móviles trienales de las precipitaciones de cada año hidrológico.

Analizando dicha media móvil, los periodos más severos de sequía han sido:

- Años hidrológicos 1941-42 a 1944-45

- Años hidrológicos 1979-80 a 1982-83

- Años hidrológicos 1990-91 a 1994-95 
En la actualidad estamos iniciando un posible período de sequía, que es más notorio debido al modelo de desarrollo económico que disfruta o padece nuestro país. Al contrario que en el caso de las crecidas, los efectos de una sequía son difíciles de evaluar o de cuantificar, entre otras razones por la dificultad de llegar a un consenso sobre la caracterización de este fenómeno o su duración. Por ejemplo, la AEMA señala como periodos de sequía que afectaron a la mayor parte de Europa el año 1971 -que no está incluido en los periodos severos que aquí se muestran- y el periodo 1988-1992, que estaría incluido sólo en parte. Desde esta perspectiva podemos diferenciar:

- Por periodo de sequía se entiende un intervalo de tiempo anormalmente seco lo suficientemente prolongado para ocasionar una disminución apreciable en el caudal de los ríos, nivel de los lagos y/o un agotamiento de la humedad del suelo, así como un descenso en los niveles de aguas subterráneas por debajo de sus valores normales.

- Entre sus efectos principales se incluyen: problemas de abastecimiento de agua, restricciones, deterioro de su calidad, efectos en la producción de energía eléctrica, pérdida de cosechas y ganado, contaminación de los ecosistemas de agua dulce e, incluso, extinción regional de especies animales.

- Las sequías prolongadas o recurrentes contribuyen a la desertización en zonas donde existe sobreexplotación de recursos hídricos, alteraciones y agotamiento de la vegetación natural, reducción de la infiltración de agua en el suelo e incremento de la escorrentía superficial que contribuye a la erosión del suelo. Los países mediterráneos son los más susceptibles de sufrir la desertización, en particular las zonas semiáridas de morfología montañosa, pendientes abruptas y períodos de fuertes precipitaciones que aumentan la erosión.

En tercer lugar, debemos hacer referencia a los incendios forestales. En el lapso que se extiende desde 1994 hasta la actualidad, nos encontramos ante un tema que, lamentablemente, ha cobrado un gran protagonismo en países como Portugal o España, todo ello muy relacionado con el período de sequía. De hecho, hasta éste, la superficie incendiada más afectada es la no arbolada, que se corresponde generalmente con terrenos cubiertos de matorral o pasto con una cubierta arbórea más bien baja.

Si se excluye el año 1994, en el que se quemaron cerca de 438.000 ha, la media de superficie forestal total incendiada en el periodo 1992-2011 ronda las 100.000 ha anuales. Además, en 1994 se invierte la situación anterior, ya que en ese año se quemó más superficie arbolada que no arbolada (conviene destacar que ese año se caracterizó por ser un año de sequía). En el período 1991-2011, el mayor número de incendios producidos se encuentran en la categoría de intencionados (60\%), siendo esta causa responsable de casi la mitad de la superficie incendiada en dicho periodo. 
Los incendios debidos a causas naturales (rayos) representan sólo el $3,8 \%$ del total, afectando al $10,6 \%$ de la superficie forestal incendiada. Desde 1978 se observa una disminución de la superficie media de los incendios. En ese año se produjo el valor máximo, con más de 50 ha por incendio frente a las 5,4 ha del año 2002, debido, muy probablemente, a la eficacia de los medios de extinción (a pesar de lo cual, no siempre se ha podido evitar las pérdidas de vidas humanas).

En cuarto lugar, nos encontramos con los accidentes por carretera y ferrocarril con emisión de sustancias peligrosas. Y es que, el transporte de mercancías por carretera es responsable de la mayoría de los accidentes con emisión o vertido de sustancias peligrosas. El transporte de mercancías peligrosas por carretera es el que presenta un mayor riesgo en número de accidentes, comparado con el transporte de mercancías por ferrocarril y por tráfico marítimo. En el año 2011 se produjeron más de 75 accidentes por carretera, frente a los 4 de la red ferroviaria y los 7 del transporte marítimo.

En quinto lugar, respecto de los accidentes marítimos con vertido de hidrocarburo destacar los vertidos procedentes de accidentes marítimos causan grandes daños en los ecosistemas litorales por el enorme volumen de las sustancias que se transportan y por la dificultad de su control y mitigación En el transporte marítimo de mercancías peligrosas los accidentes de los buques petroleros -junto con los que transportan sustancias químicas- son los que generan mayores daños para el medio ambiente. Los vertidos de hidrocarburos provocan grandes daños en los ecosistemas marinos, afectando a todos sus aspectos. Además, los procesos y operaciones de limpieza pueden ser muy agresivos para los hábitats, la fauna y la flora, siendo, sin embargo, necesarios debido a que la recuperación natural es muy lenta.

En el período 1991-2011 se han producido en las costas españolas 225 accidentes de petroleros que han provocado el vertido de algún tipo de hidrocarburo: petróleo, asfalto, fuel-oil, gas-oil, gasolina, nafta, gases licuados, mezclas oleosas, etc. Por Comunidades Autónomas, Andalucía y Galicia son aquéllas en las que se ha producido el mayor número de accidentes, seguidas de Canarias y Cataluña. Las consecuencias de estos accidentes pueden extenderse a otras Comunidades Autónomas e, incluso, a otros países.

Entre las principales causas de estos accidentes se puede señalar la antigüedad y el mal estado de muchos de los buques que realizan el transporte, que hace que presten servicio sin las medidas actuales de seguridad, como es el doble casco. A estas causas debe añadirse las circunstancias meteorológicas y geográficas adversas que pueden existir y la intensidad del tráfico marítimo en zonas determinadas, como es el paso del Estrecho y todo el Mediterráneo en general. 


\section{CAMBIOS EN LOS COTEXTOS DEL DESARROLLO}

De los geógrafos se espera que den respuestas de geógrafos. Nada más normal a la hora de tratar sobre el período que estamos viviendo en los momentos actuales -los prolegómenos del siglo XXI-; podríamos afirmar sin temor a equivocarnos, que uno de los aspectos que le caracterizan es el de un creciente $y$, en ocasiones, desmesurado y no siempre controlado desarrollo tecnológico, de cuyos efectos hemos empezado a tomar conciencia. Una de las causas ha sido la consideración de las anomalías y variantes que, como consecuencia del referido fenómeno, se están produciendo en nuestro entorno; todo ello resumido en la creciente preocupación por el medio ambiente, en el contexto de la Geografía Regional.

Lejos de ser este un problema circunscrito a un determinado ámbito, el que nos preocupa es prácticamente multisectorial e interdisciplinario, por lo que es difícil que alguien se sienta ajeno o desvinculado del mismo (y menos como geógrafos que somos). Por contra, todo parece indicar que la necesidad de cuidado y mejora de nuestro entorno es un derecho y un deber que todos, individual y colectivamente, debemos asumir como un reto, sobre todo pensando en las generaciones futuras.

El hombre siempre ha tendido a forzar a la naturaleza, a derivar en beneficio propio los flujos de energía que coadyuvan al funcionamiento de los ecosistemas. No contento con satisfacer sus propias necesidades energéticas metabólicas, ha tendido siempre a aumentar el consumo de energía externa -no metabólica con la que transformar y organizar el mundo a su conveniencia inmediata. De hecho, a más energía externa disponible, más poder, y -por ende- mayor capacidad competitiva. Esta no es una característica del pensamiento científico, sino del comportamiento humano. Si el hombre es más capaz que otras especies de actuar intensamente sobre el medio, esto debiera atribuirse a su poder de raciocinio-del que la ciencia es una consecuencia-, y no a la ciencia en sí misma. La crítica genérica a la ciencia y a la técnica es, en el fondo, una crítica a la capacidad de pensar, y como tal sería muy discutible (Tarrades, 1990).

De un lado, cabe argumentar que nos encontramos ante una apuesta de alcance bipolar por parte de una nebulosa denominada "terrorismo internacional" que parece perseguir dos, en primer lugar, la globalización de su empeño, en el sentido de que nadie ni nada sea invulnerable; en segundo lugar, consolidarse como único poder alternativo -aunque sólo lo sea para la destrucción.

En la actualidad la aldea global ha sido sustituida por el mercado global, dando lugar al tratado tema de la globalización, que, en su actual modalidad neoliberal, tiene carácter excluyente tanto de continentes enteros, los más subdesarrollados, como de sectores cada vez más amplios de los países desarrollados, los que no se adaptan a la permanente renovación tecnológica. Así, podemos plantearnos el siguiente interro- 
gante: ¿por qué los modelos de desarrollo regional? "Es incontestable", afirma Enzensberger, "que el mercado mundial, desde que dejó de ser una visión lejana y se convirtió en realidad global, fabrica cada vez menos ganadores y más perdedores, y eso no en el Tercer Mundo o en el Segundo, sino en los altos centros del capitalismo. Allá son países y continentes enteros los que se ven abandonados y excluidos de los intercambios; aquí son sectores cada vez más grandes de la población los que, en la competencia cada día más grande por las calificaciones, no pueden seguir y caen".

Desde esta perspectiva, los modelos de desarrollo regional pueden servir al doble reto de aproximar al desarrollo los países subdesarrollados, y encontrar un equilibrio entre globalización y regionalización. En este sentido, deben convertirse en un coadyuvante más, al servicio de los Estados, regiones,..., para superar las diferencias Sur-Norte, colocar la economía al servicio del desarrollo integral de las personas y los pueblos, denunciar el carácter idolátrico del capital, defender la democratización de los Estados en torno a los valores comunitarios,... (Tamayo-Acosta, J.J. 1999). ¿Qué repercusiones territoriales tendrán tal o cual acontecimiento? Las respuestas son multidimensionales, en una ciencia -la Geografíapoliparadigmática. Tras los acontecimientos de septiembre último, la globalización vuelve a mostrarnos la idea según la cual es un negocio de Gobierno, más que un gobierno de los negocios. Las divisiones que separan al mundo, tanto entre países pobres y países ricos, como dentro de estos últimos, parecen aún menos aceptables. Se comprende mejor que la globalización también es un discurso retórico de legitimación de las ganancias de los vencedores -tanto entre naciones como en el seno de las mismas- y que con frecuencia sirve los intereses de un corporativismo de ricos (más que el mérito comparado de cada uno, es la estructura del mundo -descendiendo hasta el específico de la Universidad- la que permite a unos ganar y a otros perder).

Dar respuesta a interrogantes como los anteriores, nos obliga a plantearnos la base conceptual del "subdesarrollo", o por alusión su antónimo "desarrollo". La revisión de la ya abundante literatura especializada conduce a un sinnúmero de definiciones que más que enmarcar el fenómeno lo que hacen es caracterizar o tipificar alguna o algunas de sus manifestaciones, las más de las veces con criterios productivistas ajenos, en general, a la inseparable dimensión social que acompaña a las variables económicas. Los países occidentales han utilizado con frecuencia su supremacía y el poder de persuasión que da el dinero, o que emana de las propias estructuras, para explotar a los países pobres o para mantener en el poder, en ellos, a regímenes corruptos.

Como señalan distintos estudiosos, es obvio que todos intuimos lo que es el subdesarrollo, pero nadie -acabamos de apuntarlo- to ha definido con precisión. Está claro que no es sólo una situación económica deprimi- 
da, y mucho menos un estadio cultural atrasado, pues dentro de la extensa gama de países incluidos en esta categoría, los hay con culturas milenarias riquísimas, y tampoco parece que todos los países desarrollados puedan darles lecciones en este terreno.

Es indudable, en cambio, que la condición de país subdesarrollado lleva consigo una situación de dependencia -política, económica, técnica,...- de otra potencia o de un grupo económico extranjero; supone también, en muchas ocasiones, una falta de participación política de su población en las tareas de gobierno, unas veces porque carece de preparación suficiente y otras porque se le impone una forma de administración sea paternalista o tiránica, que la excluye de ella. El subdesarrollo lleva implícito, desde luego, un nivel de vida extremadamente bajo, un predominio de población rural, un alto grado de analfabetismo, de paro total y paro encubierto; hambre y malnutrición crónicas, por tanto, a veces en grado muy difícil de imaginar, que hacen casi imposible el trabajo intenso y continuado: alta mortalidad infantil, morbilidad muy acusada, mala situación de la mujer -discriminada y considerada inferior- $y$, en ciertos países como es de sobra conocido-, una administración incompetente, arbitraria, venal y corrompida.

Las nociones de desarrollo y subdesarrollo corresponden a la óptica occidental, materialista -trátese de capitalismo y comunismo-, que da prioridad a los temas de carácter económico. Desde este punto de vista la diferencia entre unos y otros países es palmaria e innegable. Pero, desde el lado de los países subdesarrollados, ¿podemos decir que comparten los puntos de vista de la población de los países industriales? Indudablemente, no se puede contestar de un modo simplista, pero puede creerse que una buena parte de sus habitantes miran las cosas y la vida con ojos muy distintos. Insistimos una y mil veces en que no es sólo lo económico lo que caracteriza el subdesarrollo de los pueblos y las regiones, aunque quizá, a pesar de sus enormes dificultades, lo económico es lo más fácil de detectar y medir.

El tema del subdesarrollo es, ante todo y esencialmente, profundamente humano: tres cuartas partes de la humanidad padecen sus consecuencias, y eso se refleja en todos sus aspectos demográficos. Por todos los lados del análisis de las características de la población mundial -tasas de natalidad y mortalidad, índices de crecimiento, estructura por edades, composición profesional de la población, esperanza de vida, condiciones de la mujer, grado de instrucción, renta per cápita,...- encontraremos -con todas las gradaciones y matices que dan las diferencias entre las poblaciones de los países ricos y las poblaciones de los países pobres, entre los países desarrollados y los subdesarrollados. Es lógico -lo contrario sería imperdonable- que el interés humano del tema -es la vida de millones de otros hombres lo que está en juego- atraiga no sólo la atención de los científicos, resultando muy doloroso comprobar -aunque nadie que conoz- 
ca un poco la historia puede sorprenderse- cómo las grandes potencias emplean a estos países como peones, y cabezas de puente, en el trágico juego de la estrategia universal, condicionando su ayuda a que se muevan según su conveniencia.

Desde la perspectiva del desarrollo regional, los intereses se plasman en unos modelos que reflejan la historia del siglo XX, y más concretamente tras la Segunda Guerra Mundial, lo que se conoce como el círculo fordista basado en la producción de masas y de consumo de masas. Más aún, se podría plantear que la fase de acumulación del capital en esa época se enmarca forzosamente en una forma concreta que toma el Estado -el Estado de Bienestar-, el sindicalismo reivindicativo y el crédito al consumo. Estos factores permitieron así la relación entre producción y consumo, que fueron esenciales en la explicación del modelo de desarrollo de los años de oro.

No obstante, a partir de los años setenta los distintos análisis han señalado los cambios profundos en los resultados del modelo de crecimiento : el avance del desempleo, la precariedad y la exclusión se convertirían en los aspectos más importantes y no sólo en los países no industrializados, sino de los industrializados. Como señalan distintos informes, éstos tuvieron altas tasas de crecimiento económico entre los años 1973 y 1987, pero también se conocieron las mayores tasas de desempleo. El motivo es que la mayor parte de la producción de los países industrializados procedió de incrementos de la productividad total y el resto de un aumento de inversiones de capital, sin que se crearan nuevos puestos de trabajo.

La crisis de la acumulación en esta fase ha llevado así a una cierta reestructuración financiera en las actividades económicas en general y un reajuste en la relación empresarios/asalariados.

Es un hecho el que quizá deberíamos recurrir a una síntesis actualizadora, seleccionando de cada teoría tratada lo que aún pensemos que sigue siendo válido o útil. Somos, no obstante, conscientes al llegar a este punto que son muchos los interrogantes que quedan sin una contestación. ¿Por qué ninguna teoría ha sido capaz de darnos las soluciones que coadyuvaran a la erradicación del subdesarrollo? ¿Las interpretaciones que se han realizado, no son correctas? ¿Cuál es el papel a desempeñar por éstos?

La aproximación al tema tratado pasa por la puesta en práctica de destintos modelos de desarrollo regional que, desde la diversidad ideológica, permita superar el "monolitismo" del modelo en la actualidad vigente, y cuyas características estamos estudiando, marcándonos "a priori" la necesidad y utilidad de los susodichos modelos.

Lo que se está poniendo sobre el tapete no es un debate económico sobre el tema del desarrollo, sino uno de carácter político en el que sobresalen dos cuestiones: si nos consideramos tan satisfechos con lo conse- 
guido en la búsqueda de la superación de la desigualdad, como para eliminarla de las prioridades públicas, al menos, en cuanto a la intensidad de los recursos dedicados, $y$, en segundo lugar, qué ha cambiado en nuestra sociedad para que se esté dando esa resistencia por parte de los ricos a la solidaridad pública. Descartando, por tanto, un regreso al enfrentamiento, al odio, como estrategia, es evidente que deben buscarse modelos de desarrollo que coadyuven a la superación de los problemas descritos; este análisis conduce a reconocer la necesidad de una política de desarrollo regional, pues un desarrollo limitado a ciertas regiones presenta un doble inconveniente: por una parte, deja sin emplear o deficientemente utilizados los recursos materiales y humanos de las regiones pobres; por otra, suscita en las regiones más ricas y desarrolladas una ley de rendimientos decrecientes.

Como hemos expuesto con anterioridad a la hora de tratar de los modelos de desarrollo nos lleva hasta los elementos que sirven de base para confeccionar una teoría de desarrollo, entendiendo las teorías como hipótesis que nos permitan entender los orígenes, procesos y consolidación de las disparidades regionales, para que "a posteriori" puedan aplicarse los modelos que favorezcan el referido desarrollo regional. Los orígenes como señala Lázaro, J (1977) probablemente debamos buscarlo en el papel que las teorías clásicas han asignado al comercio internacional como difusor del desarrollo, basándose en el supuesto de la movilidad de los factores productivos y de bienes y servicios, llegándose a la conclusión de la tendencia casi determinista a un nivel de desarrollo similar en todos los países; sin embargo, la realidad ha desmentido tan optimistas e irreales previsiones.

Sin duda, de la superposición de éstos y de otros enfoques no mencionados, surgen interpretaciones que intentan comprender y explicar por qué unas regiones se desarrollan y otras no. Pese a todo de lo que no nos cabe duda en la actualidad es que el equilibrio territorial y regional alcanza particular importancia, sobre todo cuando se comprueba que en no pocas regiones subdesarrolladas la distribución de la riqueza y de la renta es más injusta que en las desarrolladas. Es por esto por lo que, si la opción por eliminar los desequilibrios tiene sentido es como instrumento para potenciar una redistribución de la renta entre las personas, junto con unos mejores y más justos niveles de bienestar y calidad de vida. Los modelos de organización y desarrollo regional se convierten de esta manera en un instrumento más para facilitar la consecución de los objetivos últimos de una transformación más justa de la sociedad. Pese a todo, debemos de ser conscientes de que la transformación de una determinada organización del espacio regional mediante la aplicación de estos modelos, se encuentra con no pocas resistencias al cambio, convirtiéndose en un objetivo a alcanzar a medio o largo plazo (quizá como alternativa para quienes echaban en falta una nueva geopolítica, tras el fin de la 
bipolaridad y su tranquilizadora geometría a dos, éste puede ser el esbozo de una futuras líneas de fuerza planetarias; el mundo se mueve hoy, agitado, en busca de un nuevo mapa geopolítico de sí mismo).

Pocos autores han sabido reflexionar sobre este tema como Sergio Boisier; sin embargo, vamos a seguir los análisis del profesor Elies Furió (1994), para quien la idea del desarrollo local es presentada fundamentalmente bajo dos acepciones. Ambas recogen algunos de los elementos esenciales propios de la nueva dinámica económica contemporánea, pero también muestran ciertos sesgos. En un primer momento, el desarrollo local significaría, esencialmente, el desarrollo partiendo de la base frente al desarrollo desde la "cúpula", el deseo de cortar las lógicas dominantes que conducen a una desestructuración de los territorios y de las solidaridades locales.

En un segundo momento, el desarrollo local se inclinaría hacia una apología de la salida de la crisis mediante la pequeña y mediana empresa. Esta concepción comparte con la anterior el ideal del desarrollo desde la base, pero éste es interpretado en su "primer grado": al mostrarse la creación de nuevas pequeñas empresas en la base, éste proceso se denominará "desde abajo". Tanto en un caso como en el otro, estas ópticas pueden conducir a callejones sin salida de no percibir correctamente la profundidad de las mutaciones de la técnica y la organización de la producción, de la división espacial del trabajo y de la propia actuación pública (Greffe, 1988)

Desde este punto de vista, el desarrollo local es interpretado como el resultado del deseo de vivir, trabajar y decidir el destino de la propia comunidad territorial, la necesidad por parte de cierto número de agentes sociales y de poderes públicos locales de responder al desafío del desempleo y la confianza en las pequeñas dimensiones empresariales. Estas preocupaciones adquieren sentido si se recuerdan algunas de las manifestaciones de las últimas crisis económicas; la desaparición de la confianza de la contribución exitosa de las grandes empresas respecto a las posibilidades de desarrollo local, la evidencia de la dependencia frente a las decisiones tomadas en centros lejanos, la pérdida o desaparición de las producciones locales,... Por tanto, al desarrollo realizado desde arriba se opone el realizado desde la base; a las lógicas aterritoriales de la economía capitalista se opone el interés local; a una lógica del beneficio que parece ser destructora se contrapone el deseo de satisfacer las necesidades de los consumidores y de los trabajadores, aunque esto implique el recurso a formas de producción diferentes o alternativas.

Los partidarios de este esquema han admitido siempre que implicaba riesgos. En territorios desigualmente provistos de recursos, estrategias de desarrollo desde la base podían conducir a estimular disparidades naturales o creadas artificialmente; y es que, el desarrollo local no ha conseguido nunca librarse de las acusaciones de autarquía. Al admitir de entrada 
la posibilidad de que se solucionasen localmente las necesidades a través de la producción, el desarrollo local dejaba creer que se podía vivir al margen o, incluso, fuera de la división internacional del trabajo con todo lo positivo que ésta implica; también se olvidaban las consecuencias negativas que sobre la economía local puede tener una mala inserción en la división interespacial del trabajo.

No debemos olvidarnos de que a este sesgo autárquico corresponde un medio rural; la cuestión de una cierta protección frente a los problemas de la división internacional del trabajo y de un cierre local de la economía corresponde mejor, de entrada, a medios rurales que a medios urbanos, en los que resulta difícil protegerse de la división internacional del trabajo. Este planteamiento en favor de lo "rural" y en detrimento de lo "urbano", no ha contribuido a reforzar el carácter innovador del desarrollo local. Y, también, a subestimar la problemática que puede encerrar una base productiva agraria deficiente (Greffe, 1988)

Un último problema es la falta de un mínimo de articulación entre el ámbito local y el ámbito estatal. En muchas ocasiones se ha pensa que era posible el desarrollo local al margen de las regulaciones definidas a nivel nacional. Es cierto que algunas de las modalidades de regulación estatales se muestran ineficaces para resolver ciertos problemas; aunque no está claro que esto se resuelva mediante la regulación regional y local. Pese a todo, sería erróneo concluir de lo anterior la escasa oportunidad del concepto del desarrollo local y de una estrategia fundamentada en el mismo, como un elemento básico para un mejor entendimiento de los modelos de desarrollo regional; pues, esta estrategia del desarrollo local ha demostrado, más que nunca, que la movilización de las fuerzas y su coordinación mediante proyectos colectivos es una condición "sine qua non" del desarrollo en un período de reestructuración. Iniciar una concertación, clarificar los proyectos de unos mediante los otros, y reforzar así sus posibilidades de realización, esto significa también obligarse a elegir interlocutores, definir terrenos de concertación y correr el riesgo de sacar a la luz intereses que no tienen por qué converger.

Aunque sea ésta la aportación más destacada, también se puede deducir de ella otra consecuencia: "a pesar de que no sea un modelo, el desarrollo local es, como mínimo, un método para aproximarse a los problemas" (Greffe, 1988); o, como expresan otros autores, esta perspectiva es ante todo una aproximación territorial al desarrollo. Parafraseando al profesor Vázquez Barquero (1998), la conceptualización de desarrollo local se puede relacionar con la confluencia de dos líneas de investigación: una, más bien de carácter teórico, que nace como consecuencia del intento de encontrar una noción de desarrollo, que permita la acción pública para el desarrollo de localidades y regiones; otra, de carácter histórico, que surge como consecuencia de la interpretación de los procesos de desarrollo industrial en localidades y regiones del sur de Europa. La capaci- 
dad de liderar el propio proceso de desarrollo, unido a la movilización de los recursos disponibles en el área, de su potencial de desarrollo endógeno, conduce a una forma de desarrollo que ha venido en denominarse desarrollo endógeno o desarrollo local. Este concepto de desarrollo reúne un conjunto de rasgos y características, que le dan una configuración específica. Ante todo, hay que decir que el desarrollo local hace referencia a procesos de acumulación de capital en localidades y territorios concretos. La disponibilidad de una oferta de mano de obra, suficientemente cualificada para las tareas que realiza, y poco conflictiva, unido a una capacidad empresarial y organizativa, fuertemente articulado a la tradición productiva local y a una cultura atenta a las innovaciones al cambio y favorece la acumulación de capital en los sistemas productivos locales.

Se trata de procesos de desarrollo difuso, que se caracterizan por la organización sistémica de las unidades de producción, que permite a las empresas locales competir en los mercados nacionales e internacionales. Los procesos de desarrollo endógeno se producen gracias a la utilización eficiente del potencial económico local que permiten las instituciones y mecanismos de regulación que caracterizan a cada territorio. La forma de organización productiva, las estructuras familiares y tradiciones locales, el tejido social y cultural y los códigos de conducta de la población condicionan los procesos de desarrollo local, favorecen o limitan la dinámica económica y, en definitiva, determinan la "senda" específica del desarrollo de las economías locales y regionales.

Además, el desarrollo local endógeno obedece a una visión territorial (y no funcional) de los procesos de crecimiento y cambio estructural, que parte de la hipótesis de que el espacio no es un mero soporte físico de los objetos, actividades y procesos económicos, sino que es un agente de transformación social. Cada territorio se vincula al sistema de relaciones económicas de un país en función de su especificidad territorial, de la identidad económica, política, social y cultural.

El concepto de desarrollo local endógeno concede un papel predominante a las empresas, a las organizaciones, a las instituciones locales, y a la propia sociedad civil, en los procesos de crecimiento y cambio estructural. Es una aproximación «de abajo-arriba» al desarrollo económico, que considera que los actores locales, públicos y privados, son los responsables de las acciones de inversión y del control de los procesos. Puede decirse que el desarrollo local endógeno es un proceso de crecimiento económico y cambio estructural, que conduce a una mejora del nivel de vida de la población de la localidad, en el que se pueden identificar al menos, tres dimensiones: una económica, caracterizada por un sistema específico de producción que permite a los empresarios locales usar, eficientemente, los factores productivos y alcanzar niveles de productividad suficientes para ser competitivos en los mercados; otra sociocultural, en que el sistema de relaciones económicas y sociales, las instituciones loca- 
les y los valores sirven de base al proceso de desarrollo; y otra política y administrativa, en que las iniciativas locales permiten crear un entorno local favorable a la producción e impulsar el desarrollo sostenible.

Desde esta perspectiva, frente al modelo de crecimiento concentrado y desarrollo desde arriba, tal y como hemos expuesto, ha ido definiéndose en los últimos años el paradigma de desarrollo autoconcentrado y difuso, basado en la utilización productiva de los recursos locales. Al menos en teoría, las comunidades territoriales disponen de un conjunto de recursos (económicos, humanos, institucionales y culturales), que constituyen las potencialidades de desarrollo endógeno de un área. En el entorno local se detecta, por ejemplo, la dotación de una determinada estructura productiva, mercado de trabajo, capacidad empresarial, recursos naturales, estructura social y política, o tradición y cultura, sobre la que puede articularse su crecimiento económico y la mejora del nivel de vida de la población.

Es importante subrayar que la atención prestada a los modelos locales de desarrollo no es sólo el resultado de la diferenciación de situaciones económicas locales y la emergencia de nuevas formas de industrialización y desarrollo, sino, también, es una consecuencia de la crisis del "paradigma funcionalista" de desarrollo, que consideraba al espacio como un simple lugar donde ocurrían los efectos de los procesos de desarrollo general.

Los actores ocupan un lugar central, empero este reconocimiento es insuficiente para entender el desarrollo local. El análisis no debe ser una mera exposición de las estrategias de adaptación de los actores considerados individualmente o en grupo; por el contrario, es preciso analizar y entender las relaciones específicas que vinculan a los actores entre sí; las redes humanas de relaciones entre actores existen siempre, pues son la expresión de la vida social, siendo conscientes de que estas redes no son siempre dinámicas y el medio del que forman parte puede bloquear su capacidad de renovación.

De todo ello se deriva el hecho de que en los últimos tiempos los modelos de desarrollo se encuentran sometidos a dos tipos de reflexiones. Por una parte, de naturaleza tanto descriptiva como teórica, sobre la existencia de una heterogeneidad de modelos de desarrollo y la convicción creciente sobre la necesidad de seguir caminos diferentes de desarrollo. Y, por otra parte, la reflexión acerca de la recuperación de la dimensión territorial en el análisis de los procesos económicos.

Las estrategias de desarrollo diseñadas a partir de las teorías tradicionales vienen siendo objeto de un progresivo abandono, debido principalmente tanto al fracaso de los esquemas de política interregional a nivel nacional para la corrección de los desequilibrio territoriales, como a su falta de capacidad para dar explicación a la emergencia de patrones autónomos de desarrollo en muchas regiones relativamente periféricas (Garafoli, 1992). Por otro lado, se está dejando notar la cada vez más 
débil movilidad de las empresas (capital) y de la mano de obra (trabajo). elemento clave sobre los que se basaban los enfoques tradicionales.

Estos hechos han ocasionado que se venga produciendo un giro importante en la interpretación del desarrollo regional analizado en apartados anteriores, en favor de un grupo de novedosas aportaciones -de diversa naturaleza, pero con elementos comunes- que siguiendo a sus exponentes más destacados, convendremos en llamar "Enfoque del Potencial Endógeno". En este apartado trataremos de mostrar, en forma sintética y que equipara el fundamento de esta orientación teórica, con ramificaciones tan dispersas que hay quien prefiere hablar simplemente de "tentativa de un nuevo paradigma" o de experiencias aisladas, más que de una "teoría" del desarrollo endógeno.

Indudablemente, tras lo hasta aquí expuesto comprobamos que si todas las teorías se nos han presentado en algún momento como directrices a seguir para el logro del desarrollo, en la actualidad se presenta como el eje conductor la relacionada con los modelos de desarrollo local y el desarrollo endógeno; sin embargo, "per se", no llegan a explicar de forma convincente la problemática con la que nos enfrentamos.

Como es lógico, a la hora de confeccionar las distintas fichas, relativas al modelo que se ha seguido o se aplica en un territorio concreto -estado, región,...-, debemos seleccionar aquellas cuestiones que nos parezcan más relevantes, valorando las ideas y cuestiones que puedan llegar a caracterizar dicho modelo, siendo conscientes que la selección de una variables, en no pocos casos, puede entrañar la exclusión de otras (para ello, puede ser de notable interés partir de una bibliografía lo más objetiva y amplia posible). Antes de detenernos en algunos ejemplos concretos queremos significar con el profesor F. Díaz Pineda que en la actualidad se habla, principalmente del modelo de desarrollo sostenible, "sin embargo, no hay este tipo de modelos en ninguna región del mundo. Hay más de treinta definiciones de este concepto, pero no existe ninguna puesta en práctica. Me parece a mí que por dos razones. Primero porque el concepto de desarrollo sostenible no se puede poner en práctica en una provincia dentro de un país o dentro de un país olvidando los de toda una gran región. Esto generaría un problema de competencia importante. Y segundo, porque la democracia participativa es una cosa bastante ausente en la mayoría de los países. Las opiniones de la población en materia de medio ambiente tardan realmente mucho en conocerse y sopesarse para que se lleven a la práctica por parte de la administración"'" para añadir, posteriormente, "Aunque he dicho antes que no conocía ejemplos que pudiesen considerarse como extrapolables de desarrollo sostenible, he defendido en otros casos que justamente la cultura mediterránea, por resultarme más inmediata, vivir en ella y conocerla relativamente bien, creo que ofrece, si no ejemplos de aquel concepto, al menos sí marcos excelentes para esta idea (me estoy refiriendo en general a muchas culturas rurales, mile- 
narias, a los ejemplos vivos que hay de conservación de recursos de una manera verdaderamente sostenible). Pero, claro, el desarrollo sostenible tendría que aportar un modelo que fuese extrapolable a todo el mundo, de la misma forma que lo ha sido ese otro concepto de desarrollo no sostenible que está dando lugar a tantos problemas ambientales. Se cuenta hoy con una tecnología más capaz que la tradicional, pero falta el arraigo de una conciencia ambiental.

Entre otros objetivos, un modelo de desarrollo sostenible debe conservar la diversidad, la diversidad de paisajes, la diversidad de cultura y la diversidad biológica. Curiosamente, en el mundo desarrollado la gente está perdiendo la cultura de la diversidad. Antes, Emest von Weizsáker y William Clark se han referido a otros tipos de diversidad. A mí me consta que el ama de casa cuando va al mercado termina comprando una lata de almendras de determinada marca convencida en su subconsciente de la actual capacidad de la tecnología que lo resuelve todo: la máquina que fabrica las almendras debe funcionar perfectamente porque todas las almendras son iguales. Ha perdido la cultura mediante la cual, habíamos aprendido a cultivar muchísimas razas y variedades de almendras. Y lo mismo ocurre con otros productos alimentarios. Cuando recorres el campo, encuentras docenas y docenas de variedades de manzanas, almendras, trigos, etc., que se están perdiendo porque el mercado impone sus reglas"

El desarrollo sostenible debería inspirarse en algo ya viejo bajo el sol y que quizás no sea perfecto (el hombre tecnológico lo consideraría así porque representa un ejemplo de la existencia de circuitos de regulación local de la economía), pero que puede ser complementado eficazmente con la moderna tecnología" (Díaz Pineda, F. 1996).

\section{TRANSFORMACIONES EN LOS MODELOS, CON BASE EN CATÁSTROFES AMBIENTALES}

La catástrofe del Prestige - de la que se cumple el 19 de noviembre de 2012, diez años- fue el resultado de un modelo energético insostenible en el que la industria del petróleo opera a escala planetaria y minimizando costes como principal objetivo, a costa de un elevado impacto ambiental. Ejemplifica perfectamente los efectos más perversos de la globalización neoliberal sobre el medio ambiente, con especial repercusión en las áreas costeras.

El movimiento ecologista quiere destacar que todo lo relacionado con el Prestige no fue un accidente. Es el resultado de un modelo energético concreto, de un sistema económico guiado únicamente por el beneficio a corto plazo, y cuyos efectos fueron posteriormente maximizados por una Administración pública que dio la espalda al medio ambiente en todo momento.

En los tanques del Prestige, hundido a 130 millas de la costa y a unos 3.800 metros de profundidad, en una zona de cierto riesgo sísmico, están 
almacenadas aún unas 1400 toneladas que suponen una amenaza real para la costa

Según las autoridades, unas $75.000 \mathrm{Tm}$ de fuel fueron retiradas del mar y de las costas, mezcladas con algas, arena, piedras, plásticos, etc. El acuerdo entre la Xunta y el Gobierno central sobre el sistema de gestión para estos residuos poco contribuye a clarificar la solución, ya que se prevé únicamente la separación de los materiales ajenos al fuel, plásticos en primer lugar, y arena y agua después, para obtener lo que se denomina un fuel "reciclado y dispuesto para su utilización".

Los estudios epidemiológicos se iniciaron muy tarde. No se realizaron mediciones atmosféricas ni se analizaron, durante los primeros meses, los metabolitos de hidrocarburos en las personas afectadas.

Aunque ya han pasado algunos años deberían realizarse análisis permanentes alimentarios completos, especialmente en moluscos bivalvos, incluyendo HAP y metales pesados, haciéndose públicos, posteriormente. Estos análisis se deben hacer públicos.

Por otra parte, las tareas de limpieza mostraron la utilización de las máquinas hidrolimpiadoras se ha hecho en muchos casos sin respetar el protocolo de actuación del propio Ministerio de Medio Ambiente, causando un impacto global importante en las comunidades supramareales e intermareales. El Parque Nacional Marítimo-Terrestre de las Islas Atlánticas, el Parque Natural del Complejo Dunar de Corrubedo y Lagunas de Carregal y Vixán y otras zonas de alto valor ecológico propuestas para formar parte de la Red Natura 2000 (Monte y Laguna de Louro, Carnota-Monte Pindo, Costa da Morte, Costa Ártabra,...) sufrieron un importante impacto negativo adicional al causado por las mareas negras, debido a la ejecución ambientalmente inadecuada de los trabajos de recogida del fuel.

En no pocos casos, los daños al medio natural causados por estas desde su inicio fueron muy importantes, siendo escandalosa la falta de sensibilidad y el desconocimiento de los valores del medio natural, o la inhibición en su defensa, exhibido por las diversas administraciones. Es imprescindíble que los protocolos establecidos se cumplan adecuadamente por todas las personas implicadas en las tareas de extracción de fuel y que no se agraven los daños causados por la recogida de fuel. Posteriormente, se mostró imprescindible el inicio de las labores de recuperación ambiental que contrarresten los daños provocados por las tareas de limpieza: restauración de zonas dunares, eliminación de pistas de acceso y restauración ambiental de su trazado, etc.

Por otra parte, la aparente normalidad se consiguió trasladar también a la pesca. En estos cinco años hay ya indicios de efectos sobre algunos recursos concretos, como puede ser el percebe o el mejillón, donde la disminución de individuos juveniles hace prever problemas en el reclutamiento. Este problema puede estar sucediendo con otras especies. Fondos 
someros, de gran importancia para la cría de muchas especies y vía de entrada de contaminantes en la red trófica, están aún afectados por el fuel. La apertura de la actividad extractiva fue en muchos casos precipitada.

Desgraciadamente poco hemos aprendido sobre los efectos a medio y largo plazo sobre nuestros ecosistemas y nuestra economía de las múltiples mareas negras que ha sufrido España y Galicia en el pasado, la última en 1992. El caso del Prestige, dada su magnitud, debe ser un punto de inflexión en este sentido. Es fundamental que se investigue en profundidad la incidencia de la catástrofe en los ecosistemas litorales y marinos afectados, sobre todo en lo relativo a las especies protegidas que deberían estar ya sujetas a Planes de Conservación. La investigación debe incluir el impacto a largo plazo sobre su funcionamiento integral y no únicamente sobre aquellos recursos naturales de interés comercial. La investigación no se debe limitar a estudiar el impacto sobre el medio biofísico de la catástrofe, sino que debe considerar también el impacto social, cultural, económico y demográfico en las zonas afectadas.

Igualmente, es muy importante que la investigación se dirija no sólo a conocer los impactos del accidente sino que se oriente también a la recuperación de los ecosistemas y las comunidades humanas afectados. Los resultados de todas estas investigaciones deben ser en todo momento públicos.

Desde una perspectiva económica, se planteó El Plan Galicia, que de haberse aplicado en su totalidad, hubiera promovido un modelo de desarrollo ecológicamente insostenible, mostrándosenos insuficiente para garantizar la recuperación ambiental y socioeconómica de las zonas más afectadas por la marea negra y del conjunto de Galicia. El Plan Galicia, que destinó el 96,7\% de su presupuesto a infraestructuras de transporte, representa una nueva marea destructora del medio ambiente, en este caso no de fuel sino de hormigón y asfalto. La construcción de nuevas autovías y vías de alta capacidad, de trenes de alta velocidad estricta (350 $\mathrm{km} / \mathrm{h}$ ), del puerto exterior de A Coruña, de los 24 embalses previstos en el Plan Hidrológico de Galicia-Costa... provocó un fuerte impacto ambiental negativo y ha supuesto una elevada inversión económica pública, socialmente poco rentable.

Resulta paradójico que la marea negra del Prestige haya sido la causa de un Plan que apostó por un modelo de transporte energéticamente ineficiente y fuertemente dependiente del petróleo, modelo que está en el origen de las mareas negras y de otros graves problemas ambientales como el cambio climático global.

En definitiva, las Administraciónes y la sociedad deberían entender la catástrofe del Prestige como una llamada de atención sobre los efectos ecológicos y sociales de una economía insostenible y como una oportunidad para trabajar seriamente a favor del medio ambiente, dada su relación directa con el bienestar y con la calidad de vida. Por ello debería rea- 
lizarse una evaluación ambiental estratégica analítica, sino del Plan Galicia, ya superado, sí de sus consecuencias, que deberían haberse dirigido más hacia la búsqueda de un modelo de desarrollo ecológicamente sostenible y socialmente más justo, habiendo dedicado mayor atención a la protección y recuperación ambientales, al fortalecimiento del transporte colectivo (especialmente en las áreas metropolitanas), o a aumentar el esfuerzo en $\mathrm{I}+\mathrm{D}+\mathrm{i}$.

Sin embargo, no debemos olvidar que uno de los elementos más positivos en la evolución de la catástrofe ha sido el nivel de conciencia y compromiso ambiental en defensa del mar de la ciudadanía que se ha puesto en evidencia. La respuesta de los 200.000 voluntarios y voluntarias, la autoorganización de los colectivos más afectados, las manifestaciones masivas de protesta, etc. son ejemplos que desvelan una ciudadanía democrática y madura, que quiere participar desinteresada y activamente en aquellos asuntos públicos que le atañen directa o indirectamente.

Es preciso, por tanto, dar una respuesta adecuada a los niveles de conciencia y acción ambiental puestos de manifiesto en ésta y en otras crisis. Para ello es preciso desarrollar programas y materiales educativos y formativos que faciliten a la población un conocimiento más profundo de lo sucedido, que destaquen la estrecha interdependencia entre ambiente y calidad de vida, y que actúen como barreras culturales en previsión de futuras calamidades. Así mismo, es preciso apoyar la labor social y educativa de los colectivos ciudadanos y las organizaciones ambientalistas que tienen entre sus objetivos la construcción de una sociedad democrática abierta y sostenible.

\section{A MODO DE CONCLUSIONES}

Tras lo expuesto podemos colegir las conclusiones siguientes:

1) No son pocos los territorios de nuestro país, en general, y de forma concreta en Galicia, en los que los cambios en los modelos de desarrollo, con amplias repercusiones no sólo económicas, sino también sociales e incluso políticas, se están dando a partir diversas catástrofes ambientales.

2) Deberían plantearse y generarse importantes cambios en la política energética, pues resulta un despropósito que tras la catástrofe del Prestige -hace ya casi una década- se haya mantenido una política que favorezca, cuando menos, que España cumpla los compromisos asumidos del Protocolo de Kioto. Por lo tanto, debería primarse una política energética basada en el ahorro, la eficiencia y las energías respetuosas con el medio ambiente, como solución última a los impactos ambientales derivados del uso y transporte de combustibles fósiles a escala global, todo ello en paralelo a una política de transporte que potencie los modos de transporte más eficientes energéticamente y limite el uso del automóvil priva- 
do. Aspectos estos que deberían valorarse ahora que se aproxima la Cumbre de Río+20.

3) Debería acabarse con la responsabilidad ilimitada de las empresas: Como en el caso de las catástrofes de petroleros frente a nuestras costas, las industrias sucias trasladan los costes económicos de la degradación medioambiental al conjunto de la sociedad. Esta catástrofe fue el resultado también de lo "barato" que resulta contaminar y de lo fácil que es obviar el riesgo para maximizar los beneficios económicos. El hecho de que el Fondo de Indemnización Internacional para hacer frente a las indemnizaciones derivadas de la contaminación por hidrocarburos provocada por los accidentes marítimos haya ascendido de 175 a 1.000 millones de euros es un avance pero no es suficiente, el Prestige supuso a los ciudadanos unas pérdidas económicas de entre 900 y los 10.000 millones de euros.

4) Debe, por tanto, buscarse un régimen de responsabilidad ilimitada para toda la cadena involucrada en el transporte de hidrocarburos, desde el armador al propietario de la carga, incluyendo a las aseguradoras y las sociedades de clasificación. Todo buque que transporte estas sustancias debe estar en posesión de un seguro que cubra sin límite la cuantía de los daños ocasionados por un accidente. Estos costes deben incluir no sólo el impacto directo sobre los ecosistemas sino también los costes de su recuperación. Todo ello, unido a la creación de seguros que se apliquen a las áreas naturales protegidas.

5) Ha de propiciarse cambios en la política de transporte marítimo: $\mathrm{Si}$ algo puso de manifiesto la marea negra del Prestige es que en las últimas décadas la UE apenas ha avanzado en seguridad marítima. Pese a que las costas europeas han sido escenario de numerosas catástrofes de esta índole, no ha sido hasta el accidente del Prestige que las medidas incluidas en los paquetes de medidas Erika I y II han ido siendo aprobadas . Además, son claramente insuficientes para garantizar que un accidente como el del Prestige no vuelva a suceder, pero esto no va a suponer grandes cambios en el modo de operar de la industria. Por otro lado, los calendarios de eliminación de buques monocasco de estos paquetes son también insuficientes. Entre los cambios podemos destacar: la prohibición del transporte de hidrocarburos y sustancias peligrosas en general en buques de casco simple; la creación de una red de infraestructuras con todos los medios necesarios para responder a un accidente de estas características; mejorar las inspecciones; la declaración de Áreas Marinas Especialmente Sensibles (AMES). En este sentido damos la bienvenida a la propuesta realizada por 6 Estados Miembros de la UE de declarar la fachada atlántica europea, incluyendo Galicia, como AMES por parte de la Organización Marítima Internacional (OMI), aunque nos preocupa la falta de medidas acompañantes para mejorar la seguridad marítima que hagan eficaz dicha designación, así como la oposición de diversos países a que esta propues- 
ta se haga realidad. Es igualmente necesario un acuerdo en el contexto de la ONU y agencias relacionadas para resolver el problema de los pabellones de conveniencia. La UE debe jugar un papel clave en este proceso.

6) Deben realizarse nuevos Planes así como dotarse de medios de lucha contra la contaminación. Es más que sorprendente la falta de medios de prevención y lucha contra la contaminación en una de las áreas con tráfico marítimo más intenso del mundo y con un historial de catástrofes marítimas como es el caso de las costas gallegas. Una vez más, las administraciones públicas españolas han quedado en entredicho, no habiendo servido todos los accidentes anteriores para dotarse de los medios más básicos para enfrentarse a una catástrofe como la padecida. Es, igualmente, muy grave la ausencia en la práctica de protocolos o planes de contingencia, pese a sí existir en el papel. La consecuencia ha sido la ampliación del abanico de litoral afectado al arrastrar el barco siniestrado a lo largo de toda la costa gallega, alcanzando la contaminación también a las costas del Cantábrico español, Francia y sur de Reino Unido. Diez años después poco se ha avanzado en este sentido.

7) Vigilancia y prevención de contaminación difusa: Durante los últimos años se han constatado públicamente las acciones de empresas y barcos desaprensivos que aprovecharon la situación de contaminación de hidrocarburos en el mar para limpiar sus sentinas al paso por la costa o cuando se refugian de los temporales a las entradas de las rías. Estas acciones, lejos de ser puntuales o circunscritas a los últimos años, son práctica común y aportan nuevas y continuas cantidades significativas de contaminantes al medio marino. Es inexcusable un servicio de vigilancia que disuada y penalice este tipo de acciones, y que debe ser parte de un servicio integral de lucha contra la contaminación.

\section{BIBLIOGRAFÍA}

Agencia Europea de Medio Ambiente, (1998), El Medio Ambiente en la Unión Europea 1995. Informe para la Revisión del Quinto Programa sobre Medio Ambiente, Oficina de Publicaciones Oficiales de las Comunidades Europeas, Luxemburgo.

- (1998), El Medio Ambiente en Europa, Informe Dobris, Oficina de Publicaciones Oficiales de las Comunidades Europeas, Luxemburgo, (Publicado por el Ministerio de Medio Ambiente, Madrid).

- (1997), "Environmental Agreements: Environmental Effectiveness", Environmental Issues No 3, Copenhague.

- (1996), "Environmental Taxes", Environmental Issues, Series No 1, Copenhague.

AA.VV. (1991). Metodología para la Incorporación del Medio Ambiente en la Planificación Económica., Junta de Andalucía., pp.70. 
Abreu y Pidal, J.M. (1975). "El medio natural en la planificación del desarrollo"., ICONA., Madrid.

Acosta, F. (1986). "Modelos"., Revista Ecosistemas., pp. 58-59.

Aguilar, S. (1997). El reto del medio ambiente. Conflictos e intereses en la política medioambiental europea., Madrid., Alianza Universidad.

Aguilar, S. y Slocock, B.(1997). "El reto medioambiental en la Europa Oriental: Las lecciones de Occidente"., Gestión y Administración Pública., n.6. Aguiló, M., González Alonso, S., Ramos, A. (1995). Directrices y técnicas para la estimación de impactos, 39 edición, Cátedra de Proyectos y Planificación de la E.T.S. de Ingenieros de Montes, Madrid.

Alonso, J. (1990). La nueva situación regional., Madrid., Editorial Síntesis., 166 págs.

Alonso, C (1996). Variaciones sobre un mundo en cambio. Ed. Alianza. Madrid. $572 \mathrm{pp}$.

Alonso, J "et alia"(1997). Temas de Geografía de España., Madrid., UNED, $3^{\circ}$.edc. 311 págs.

Alonso, L. E. (1999). "El discurso de la globalización y la nueva desigualdad regional"., Estudios Regionales, 54., pp. 125-145.

Alonso, L .E. (1999). "El discurso de la globalización y la nueva desigualdad regional"., Estudios Regionales, 54., pp. 125-145.

Azqueta, D., Pérez, L., (Eds.) (1996): Gestión de Espacios Naturales. La demanda de servicios recreativos. McGraw-Hill, Madrid

Allende, J.(1995). "Desarrollo sostenible. De lo global a lo local"., Ciudad y Territorio-Estudios Territoriales., n. 104., pp.267-281.

Allun, P. (1995). State and Society in Western Europe., Cambridge., Polity Press.

Artigas, M (1982): Filosofía de la Ciencia Experimental Pamplona., EUNSA, Atkinson, G.(1996). "Desarrollo sustentable: teoría, medición y políticas"., ICE, n.751., pp. 15-25.

Banco Mundial (1988): Informe sobre el DesarrolloMundial Las finanzas públicas en el proceso de desarrollo. Washington.

Banco Mundial (1990): Informe sobre el Desarrollo Mundial. La pobreza. Washington.

Banco Mundial (1991): Informe sobre el Desarrollo Mundial La tarea acuciante del desarrollo. Washington.

Banco Mundial (1993 y siguientes): Informe sobre el Desarrollo MundiaL Invertir en salud. Washington.

Barbarit, L.M. «et alia».(1990): La nouvelle Vendée, voyage dans la Vendée industrielle.Edt. France-Empire. 257 págs.

Barragán, J.M.(2004). Las áreas litorales en España: del análisis geográfico a la gestión integrada, Editorial Ariel., 214 págs.

Barragán, J.M.(2005). La gestión de áreas litorales en España y Latinoamérica., UCA., 198 pp. 
Barragán, J. M "et alii" (2005). Towards Integrated Coastal Zone Management in Chile., Coastal Management, 33., pp. 1-25.

Beaufais, J.(1975). "La notion de region", en L'Europe et ses régions., pp. 267-287. Liege., Faculté de droit.

Benko, G (1990). "La cite-scientifique de I'Ile de France-Sud"., Annales de Geographie, 556., pp. 660-674.

Bradford, C. (1989): «Las nuevas formas que adquieren los mercados mundiales y la naturaleza de la interdependencia en un mercado cada vez más multipolar». Revista de la Sociedad Internacional para el Desarrollo, n.2 15, pp. 58-61.

Bertelmus, P.(1990): «Sustainable development; a conceptual Framework». Diesa Working Paper Series, New York.

Berzosa, C. (1991). Los nuevos competidores internacionales. Hacia un cambio en la estructura industrial mundial., Ciencias Sociales., Madrid.

Boira, J.V. y Souto, X.M. (1995). Percepción del medio y planificación del territorio: el caso de Vigo., Boletín del la AGE, no 20., pp. 67-80.

Boisier, S. (1995). "La modernización del Estado: una mirada desde las regiones (revoluciones, reformas, objetivos nacionales y el papel del territorio)"., Estudios Regionales, no 41., pp.15-38.

Boisier, S. (1997). "En busca del esquivo desarrollo regional: entre la caja negra y el proyecto político"., Ciudad y Territorio, Estudios Territoriales., XXIX, 112., pp. 379-397.

Blacksell, M.(1994).«Environmental Policies and Resource Management», en Blacksell, M., y Wifliams, A. M.: The European challenge. Oxford: Oxford Uníversity Press.

Brundtland, G.R. (1988): Our Common Future. TherReport ofthe Wold Commission on Environment and Development. Oxford University Press.

Cabero Diéguez, V. (1994). Contribución para la definición de una estrategia de intervención y la promoción

Cabero Diéguez, V. (1996). Los espacios naturales protegidos: el dilema entre conservación y el desarrollo local., en Dinamismos socio-económicos e (re) organizaçao territorial: procesos de urbanizaçao e de reestructuraçao productiva., Instituto de Estudios Geográficos., Universidad de Coimbra., pp. 501-510.

Cabero Diéguez, V. (2001). Espacios naturales protegidos y conservación del medio., en GIL OLCINA, A y GÓMEZ MENDOZA, J. (coord.). Geografía de España., Barcelona, Edt. Ariel., pp. 207-221.

Csatári, B (1993). "El programa Alföld: un proyecto experimental de desarrollo regional en Hungría"., en AA.VV. Autonomías y desarrollo. III Seminario Hispano-Húngaro sobre Desequilibrios Regionales., UNED., Madrid., pp. 125/152.

Castells, M. "et alia"(1986). Nuevas tecnologías, Economía y Sociedad en España., Madrid., Alianza Edit. 2 vols.

Castells, M. (1999). "El mundo según Davos"., El País., 12 de febrero, pág. 18. 
C.E.E. Primer Programa Europeo contra la pobreza (1975-1980).

C.E.E. Segundo Programa Europeo contra la pobreza (1984-1988).

C.E.E. Tercer Programa Europeo contra la pobreza (1 990-1995).

CEPAL. (1997). Resúmenes de Documentos sobre Planificación y Medio Ambiente.

Club de Bruxelles (1992). "L'Environnement dans le Marché unique europeen"., Vol.1., pp. 61-63.

Cortés, R. (1996). "El Programa Español de Desarrollo y Diversificación Económica de Zonas Rurales Objetivo 1"., Baetica., vol. 18., pp.63/72.

Curbelo, J.L. "et alia". (1989). "Los estudios regionales en el contexto anglosajón: ¿"Ciencia regional" vs. "Economía política regional"?"., Estudios Regionales, n. 23., pp. 15-27.

Chesnais, F. (1997). La Mondialisation du capital., Paris., Syros., 251 págs.

Chomsky, N. (1998). "Finanzas y silencio"., Le Monde Diplomatique., diciembre., pp. 15.

Daly, H.E. (1996). "Desarrollo sostenible y escala óptima de la economía"., en Díaz Pineda, F. (editor). Ecología y Desarrollo., Madrid., Edit. Complutense., pp. 73-84.

Departamento de Proyectos y Planificación Rural.(1994). Casos prácticos en la Planificación física y Evaluación de Impactos. Fundación Conde del Valle de Salazar, E.T.S. de Ingenieros de Montes, Madrid.

Díaz Pineda, F. (1996). "Desarrollo sostenible y escala óptima en la economía"., en Díaz Pineda, F. (edt.). Ecología y Desarrollo., Madrid., Edt. Complutense., pp. 73-76.

Enkerlin Hoeflich, E (1997). Ciencia Ambiental y Desarrollo Sostenible Internacional. Thomson Editores. $666 \mathrm{pp}$.

Escobar Gómez, G.(1994). "Evaluación de Impacto Ambiental en España: resultados prácticos"., CYTET, II (102)., pp.585-593.

Esteban Moratilla, F. (1999). "La Ordenación del territorio en la perspectiva de la construcción europea"., en Jornadas Técnicas de Debate sobre Oportunidades y problemas de la Ordenación del Territorio., Sevilla., 2426 de febrero.

Estébanez, J. (1996). "Barreras que dificultan la comprensión del espacio en la etapa de acumulación flexible" (en prensa).

FMI (2000). World Economic Outlook.

FONT, N.(1996). La europeización de la Política Ambiental en España. Un estudio de implementación de la Directiva de Evaluación de Impacto Ambiental.. Universidad Autónoma de Barcelona.

Freeman, Ch. Et alia. (1985). Desempleo e innovación tecnológica. Un estudio de las ondas largas y el desdarrollo económico., Madrid., Ministerio de Trabajo y Seguridad Social.

Friedman, J. (1979). Territory and Function., University of California Press. Furió, E. (1994). "El desarrollo económico endógeno y local: reflexiones 
sobre su enfoque interpretativo"'", Estudios Regionales, no 40., pp.97-112. García Alvarado, José Ma (1997). "Propuesta teórico-metodológica para la valoración de la calidad urbano ambiental"., Anales de Geografía de la UCM., no 17., pp. 11-26.

García Alvarado, José Ma (2000). "El modelo metropolitano madrileño: enjuiciamiento desde la perspectiva del V Programa de Acción de CE. En materia de Medio Ambiente. Apuntes metodológicos"., Observatorio Medioambiental., no 3., pp. 135-158.

García Ballesteros, Aurora (1998)., "Nuevos espacios del consumo y exclusión social"., Anales de Geografía de la UCM., no 18., pp. 47-53.

García de Enterría, E. (1997). "Economía y secularización de la política"., Madrid., $A B C$., 27 de noviembre.

Garrido, A. "et alia" (1996), "Estudio del uso y valoración del parque regional de la Cuenca Alta del Manzanares (Madrid) mediante el método del coste de viaje", en Azqueta, D. y Pérez y Pérez, L. (Eds.),

Gómez-Sal, A. (1998). "Valoración multicriterio a escala local"., Ecosistemas., n.24-25., pp. 41-47.

Goodland, R.(1997). Medio Ambiente y Desarrollo Sostenible: más allá del informe Brundtland._Ed. Trotta, D.L. Madrid. 133 pp.

González Bernández, F. (1976). "problemas ecológicos de la conservación del medio ambiente"., en AA.VV. Conservación del medio ambiente., Revista de la UCM., vol. XXV, n.105., pp.165-173.

González, M. (1997): Valoración económica del uso recreativo-paisajístico de los montes: aplicacion al Parque Natural de Monte Aloia en Galicia. Tesis Doctotal, Departamento de Economía Aplicada, Facultad de Ciencias económicas y Empresariales Universidad de Vigo.

González, E (2001). De los tajos a los embarcaderos., Barcelona., Edt. Ariel., 320 págs.

Greffe, X (1988). Descentralizar a favor del empleo. Las iniciativas locales de desarrollo., Madrid., Ministerio de Trabajo y Seguridad Social.

GREENPACE (1988). The international trade in wastes. A Greenpace inventory., Washington.

Gutiérrez, J.(1998). Redes, espacio y tiempo., Anales de Geografía de la UCM., no 18., pp. 65-86.

Hagget, P., y Chorley, J.(1967). "Models, paradigms and the new geography"., in AA.VV. Models in Geography., pp. 19/41.

Halimi, S. (1998). "El naufragio de los dogmas liberales"., Le Monde diplomatique.

Heredia, R. De (1987). "Concepto y diseño del Parque Tecnológico de Madrid"., Madrid., Heredia-Consultores.

Hiernaux, D. (1995). "La región insoslayable"., Revista Eure, 63., Universidad Católica de Chile., Instituto de Estudios Urbanos.

Higgins, B. (1995). "Regional Development Theories and Their Applications". 
Hopenhayn, M (1994). "Ni apocalípticos ni integrados. Aventuras de la modernidad en América Latina"., Santiago de Chile., F.C.E.

Instituto Tecnológico y Geominero de España.(1997). Los peligros naturales de España en 1994. Ministerio de Medio Ambiente, 63 pp.

Instituto Técnológico y Geominero de España.(1998). Guía visual para la evaluación y corrección de impactos ambientales.Ed. IGME. Madrid.

Jiménez-Herrero, L. (1989). Desarrollo y Medio Ambiente. Alianza Edt. Madrid. Jiménez Herrero, L. (1996).Desarrollo Sostenible y Economía Ecológica., Madrid., Edt. Síntesis.

Myers, N. (1989): GAL4, An Atlas of Planet Management. London, Gaia Book limited.

Morales Matos, G. (1986). El grupo humano en el espacio geográfico asturiano.

Morales Matos, G, (director) (1993). Geografía de Canarias., Prensa Ibérica., 2 tomos., Las Palmas de Gran Canarias.

Kaul, I.(1996). The Tobin Tax: Coping with Financial Volatility., Oxford U.P. Lázaro Araujo, J. (1977). "Modelos de desarrollo regional"., ICE., juniojulio.,pp.14-40.

Lee,N.(1991). Quality control in the EIA process.EIA Newsletter 6:22-23. Lillian, T «et alia» (1977): «Urbanization -Industrialization andthe Theory of Demographic Transition». Pacific Sociological Review, vol. 20. ni' 1. pp. 1 13-134.

León, C. (1994): La valoración contingente del paisaje de los parques naturales del centro-occidente de Gran Canaria, Tesis Doctoral, Departamento de Economía Aplicada. Universidad de las Palmas de Gran Canaria.

López López, Alejandro (2000). "Requisitos medioambientales para un programa de acción sobre Turismo Rural en la Comunidad Autónoma de Madrid"., Observatorio medioambiental., no 3., pp. 195-222.

Maestre, L. (1992). "Política Medioambiental en España. Estado y Autonomías"., en Revista 3Economía4., n.14., pp. 58-62.

Margalef, R. (1990): «La diversidad biológica y su evolución». Panda, n. 8. pp.4-18.

Martín, M.A., Ramos A., Velarde, M.D. (Eds.).(1994). Papeles del Centro EIA. Cátedra de Proyectos y Planificación de la E.T.S. de Ingenieros de Montes, Madrid.

Mariño, F.M. (1993). "La configuración progresiva de la Política Medioambiental Comunitaria"., C.E.C., pp. 799-835.

Math,M (1993): «Population problems: constituen of general culture in the 21 century».International Review of Education. 39 (1-2), pp.5-13.

Maurer, J.L. (1995). "La economía de Indonesia"., Rev. Estudios Asiáticos., no 1., pp. 51/78.

Magadán, M., Rivas, J., (1998), Economía Ambiental: Teoría y Políticas, Editorial Dykinson, Madrid.

Magariños, Antonio (1999). "La Información Ambiental: viejas necesida- 
des, nuevas demandas y soluciones pendientes"., Madrid., Academia de las Ciencias,..., "Homenaje a D. Ángel Ramos"., pp. 1395-1414.

Manzini, E.(1996): Artefactos: hacia una nueva ecología del ambiente artificial. Ed. Celeste. Madrid. 206 pp.

Méndez, R. "et alia" (1998). "Procesos de industrialización periférica y espacios emergentes en Castilla-La Mancha"., Anales de Geografía de la UCM., no 18., pp. 177-204.

Ministerio de Medio Ambiente. España.(1996). Seguimiento de la contaminación producida por el accidente del buque Aegean Sea. Ed. Ministerio del Medio Ambiente, Centro de Publicaciones. Madrid. 185 pp.

Ministerio de Medio Ambiente. España.(2000). Haciendo camino hacia el desarrollo sostenible en España. Ministerio de Medio Ambiente. Centro de Publicaciones. 63 pp.

Mitchel, R.C., "et alia". (1989): Using Surveys to Value Public Goods: The Contingent Valuation Method, Resources for the Future, Washington, D.C. Meadows, D.H. «et alia». (1972): Limits to Growth. New York. Universe Books. Meadows, D.H. (1996). "Más allá de los límites"., en Díaz Pineda, F.(editor). Ecología y Desarrollo., Madrid., Editorial Complutense., pp. 57-73.

Menard, S «et alia» (1987): Perspectives on population.An introduction to concepts and Issues, Oxford - New York. Oxford University Press.

Morales Matos, G. (1986) El grupo humano en el espacio geográfico asturiano., Servicio de publicaciones de la Universidad de Oviedo, Oviedo.

Morales Matos, G. (dir.). Geografía de Canarias., Prensa Ibérica., 2 tomos., Las Palmas de Gran canarias.

Mortimore, M. (1992). "El nuevo orden industrial internacional"., en Revista de la CEPAL., no 48.

Moss, R.H. «Research on global change and its human dimensions». IGBP Newsletter, 9. pp.12-15.

Muñoz, J.(1992): «Perspectiva ambiental e integración disciplinar en Geografía», Boletín de la A. G.E. n. 14, pp. 1-6.

Muñoz, R. (coord.). Crisis y futuro del Estado de Bienestar. Madrid, Alianza Universidad.

Naredo, J.M. (1987). La economía en la evolución., Siglo XXI.

Naredo, J.M. (1998). "¿A dónde va a parar la crisis?"., Le Monde Diplomatique., octubre., pág. 2.

Noin, D.(1983): La transition démographique dans le monde. Paris, P.U.F. Odum, H. (1980): Ambiente, energía y sociedad. Barcelona. Edt. Blume. 410 págs.

Paz, M.A. de (1998). Economía mundial., Madrid., Edt. Pirámide., 605 págs. Pérez Villar, M. (1990). "Perspectivas de Desarrollo Económico en Castilla y León"., en Actas del Primer Congreso de Economía Regional de Castilla y León., pp. 12-50. 
Pérez Díaz, V.(1996). Política y economía del agua en España: criterios, alternativas y proceso de aprendizaje. Madrid. Círculo de empresarios. $158 \mathrm{pp}$.

Peris Mora, Eduardo.(1997). Contaminación y uso del litoral de las costas de España. Ed. Universidad politécnica de Valencia, Departamento de ingeniería de la construcción. Valencia.

Pérez, L. "et alia" (1996a): "El Valor de Uso Recreativo del Parque Nacional de Ordesa y Monte Perdido: Coste de Viaje versus Valoración Contingente" en Azqueta, D. y Pérez y Pérez, L. (Eds.)

Pérez, L., "et alia". (1996b):"La valeur d'usage á des fins de loisir des espaces protégés en Espagne. Comparision entre méthode des cóuts de déplacement et méthode d'evaluation contingente". Cahiers $d$ 'Economie et Sociologie Rurales, no 41, pp. 40-5 6.

Poole, M.(1989): Protección del Medio Ambiente en los proyectos de gasoductos. Barcelona. SEDIGAS.

Precedo, A. (1994). Desarrollo territorial y planificación comarcal., Xunta de Galicia., Santiago de Compostela., 190 págs.

Prera Flores, A. (1998). "¿Sociedad de mercado? No, gracias"., El País., 27 de agosto.

Puyol, R. (1982): Población y espacio. Problemas demográficos mundiales. Madrid. Edt.Cincel.

Puyol, R. (1984): Población y recursos. El incierto futuro. Madrid. Edt. Pirámide.

Ramonet, Ig (1998). "El fracaso de los dogmas neoliberales"., Le Monde Diplomatique., diciembre., pág. 18.

Ramos, A. «et alia» (1979): Planificación física y ecología. Modelos y métodos. Madrid.Edt. E.M.E.S.A.

Ramos, A. (edt) (1987): Diccionario de la naturaleza. Hombre, ecología y paisaje.

Madrid. Espasa-Calpe.

Ramos, A. (coordi.) (1990): «Medio Ambiente y crecimiento económicos. Revista del Instituto de Estudios Económicos, nº 2.

Rebolledo, D., "et alia" (1994): "Valoración contingente de bienes ambientales: aplicación del Parque Natural de la Dehesa del Moncayo ", Documento de trabajo 94/6, Servicio de Investigación Agraria, Unidad de Economía y Sociología Agrarias, Diputación General de Aragón, Zaragoza. Recreo Jiménez, F.(1997).Consideración del cambio medioambiental en la evaluación de la seguridad: escenarios climáticos a largo plazo en la Península Ibérica. Publicación Técnica ENRESA. 110 pp.

Riera, P. (1994): Manual de valoración contingente, Instituto de Estudios Fiscales, Madrid. 
Riera, P., "et alia" (1994): "El valor de los espacios de interés natural en España. Aplicación de los métodos de la valoración contingente y el coste del desplazamiento". Revista Española de Economía, no monográfico "Recursos Naturales y Medio Ambiente", Pp.207-230

Rivas, David. M.(1997). Sustentabilidad: desarrollo económico, medio ambiente y biodiversidad Ed. Parteluz. Madrid.

Rodríguez Porras, Javier.(1989). Problemas de contaminación ambiental: causas y rasgos distintivos en Madrid capital. Madrid. Ayuntamiento.

Rodríguez Sáiz, L., (1988), "Política Económica y Medio Ambiente", Revista Comunidad Educativa, ICCE, No 158, Educación Ambiental, Madrid, págs.14-16.

Rodríguez, P.J. (2000). "Aproximación a los diversos Programas de Acción Comunitaria en materia de Medio Ambiente"., Observatorio Medioambiental., n० 3., pp. 455-488.

Romero, J. y Pérez, J.(1992): Pobreza y desigualdad en los países en desarrollo. Madrid. Edt. Síntesis.

Romero, J. «et alia».(1992): Desigualdades y nueva pobreza en el mundo desarrollado. Madrid. Edt. Síntesis.

Runyan, C. (1999). "La crisis de Indonesia"., Ecología World-Watch., nº 6., pp. $10 / 40$.

Ruesga, S.M. (1991): «Reflexiones preliminares sobre la evaluación monetaria del

Medio Ambiente». Rev. Situación, W 2. pp. 155-162.

Sánchez, J. "et alia" (coord.). II Seminario Hispano-Húngaro sobre desequilibrios regionales"., Madrid-La Rioja., 7-12 de octubre.

Santos, M. (1973): Geografía y economía urbanas en los países subdesarrollados.

Barcelona. Oikos-Tau.

Santos, M. (1988): «Nuevo orden internacional y reorganización espacial». Urbanización, Subdesarrollo y crisis en América Latina. Seminario de Geografía. Albacete. pp. 27-34.

Santos, M. (1993). Los espacios de la globalización., Madrid., Anales de Geografía de la Universidad Complutense., n 13., pp. 69-80.

Sauvy, A.(1973): ¿Crecimiento cero? Barcelona. Edt. Dopesa. 255 págs, Sanz, J.J. y García Rodríguez, Ma P.(1991): «Desertificación, erosión y degradación de suelos». Rev. Situación, ng 2. pp. 55-71.

Sanz Fonfría, Ramón.(1989). Ingeniería ambiental: contaminación y tratamientos. Barcelona. Marcombo Boixareu Editores, 145 pp.

Sebastián, L. DE (1988): La crisis deAmérica Latina y la deuda externa. Madrid. Alianza Editorial

Seoánez Calvo, Mariano.(1996). Ingeniería del medio ambiente aplicada al medio natural continental: la contaminación del medio natural conti- 
nental: aire, aguas, suelos, vegetación y fauna. Tecnologías de identificación, lucha y corrección. Ed. Mundi-Prensa: Análisis y trabajos prospectivos. Madrid. $701 \mathrm{pp}$.

Soler, Manuel A.(1997): Manual del Gestión del Medio Ambiente. Ed. Ariel. Barcelona. 475 pp.

Suárez , José Luis (1997). El Nuevo Plan General de Ordenación Urbana de Madrid: oportunidades para los operadores urbanos. Ed. IESE: Caja de Madrid. Ayuntamiento de Madrid. 454 pp.

Semprúm, J. (1998). Geopolítica del hambre, 1998/1999., Madrid, ACH. Sevilla, J. (1999). "Dinámica de pobres y ricos"., El País., 9 de enero., pág. 12. Shaw, R.P. (1992): «Environment Impact». Assessment Review. marzo-junio. Simón, J. (1 98 1): The Ultimate Resource. Princeton, Princeton University Press.

Simón, J. y KAHN, H.(1984): TheResourcefulEarth. New York. Basil Blackwell Inc.

Soldevilla, E. (1991). "La política medioambiental de la Comunidad Europea"., Rev. Situación., n.2., pp. 163-176.

Sotelo Navalpotro, Justo (1995)., Economía Española: Los Marcos Sectorial y Social, Editorial Mapfre, Fundación Mapfre Estudios, Instituto de Ciencias del Seguro, Colección Universitaria, Madrid.

Sotelo Navalpotro, Justo y Algarra, A.A.(1999). "Política Económica y Medio Ambiente"., Observatorio Medioambiental, no 2., pp. 311-330.

Sotelo Navalpotro, José Antonio (1998). "Los contextos de la Política Ambiental Española actual: adaptación del Quinto Programa de la U.E."., Observatorio Medioambiental., no 1., pp. 127-140.

Sotelo Navalpotro, José Antonio (1998). "Medio Ambiente y Desarrollo en la España de los noventa: la problemática regional de los residuos tóxicos y peligrosos", Anales de Geografía de la UCM., no 18., pp. 257-280.

Sotelo Navalpotro, José Antonio (1999). Modelos de Organización y Desarrollo Regional., Madrid., IUCA. 195 págs.

Sotelo Navalpotro, José Antonio (2000). Regional Development Models., Oxford University Press.

Sotelo Navalpotro, José Antonio (2000). Medio Ambiente y Desarrollo en España en los prolegómenos del siglo XXI: Las Políticas Medioambientales de la U.E.", Observatorio Medioambiental., no 3., pp. 341-397.

Sotelo Navalpotro, J.A. (2007). Environmental, Develolpment and sustainability. Contradictory models and performance policies. Oxford University Press. 428 pp. ISBN 978-84-930734-6-6

Sotelo Navalpotro, J.A.; Sotelo Pérez, M; Tolón Becerra, A. (2011). Las emisiones de Gases Efecto Invernadero en el sector transporte por carretera. Investigaciones Geográficas, no 54. 133-169 pp. 
Sotelo Navalpotro, J.A.; Tolón Becerra, A; Lastra, X (2011) Indicadores por y para el desarrollo sostenible, un estudio de caso. Estudios Geográficos. Vol LXXII, 271. 611-654pp.

Stöhr, W.B. (1986). "La politique japonaise des technolopes: Innovation Technologique et industrielle"., en Federwich, J. "et alia". "Technologie nouvelle et ruptures regionales"., Paris., Económica., pp. 123/139.

Solozábal, J.Ma. (1989). Curso de Economía., Bilbao., Edt. Deusto.

Surendran, M (1992): «Medio Ambiente y Subdesarrollo». en VV.AA. Guía de acción joven sobre desarrollo sostenible». Madrid, AIESEC.

Tamayo-Acosta, J.J. (1999). "El cristianismo en tiempos de globalización"., El País, 4 de enero, pág. 12.

Tamames, R. (1 977): Ecología y Desarrollo. Madrid. Alianza Edt.

Tamames, R. (1989).Pobreza, penuria y subdesarrollo. Documentación Social, n. 76. pp.33-41.

Toledo, V.M. 1987. Ecología y autosuficiencia alimentaria. México: Siglo XXI. Toledo, V.M. 1993. Ecología y nueva Ley Agraria en México: preludio y fuga de una modernización obsoleta. Alternativas para el Campo Mexicano. México: Fontamara.

Tolón Becerra, A; Lastra Bravo, X; Sotelo Navalpotro, J.A. (2012). Territorial distribution of transport emisión reduction targets from an environmental, economic and social viewpoint. Environmental Science \& Policy. No 16, 97-113 pp.

Torres Luna, Ma. P. "et alia". (1990). Galicia., rexión de contrastes xeográficos., Universidade de Santiago de Compostela., 210 págs.

Torres Luna, Ma.P."et alia" (Editores)(1993). Los Caminos de Santiago y el Territorio., Congreso Internacional., Santiago de Compostela., Xunta de Galicia., 962 págs.

Thedieck, R., (1963), "Vers Une Politique Industrielle", L'Observateur de I'O.C.D.E., N05, Agosto, París.

Therbon, J. (1989): «Los retos del Estado de Bienestar: la contrarrevolución que fracasa, las causas del malestar y la economía política de las presiones de cambio».

Trías, E. (1998), Entre el casino global y el santuario local., El Mundo., 1 de septiembre.

Vázquez, C. (1996). Urbanización y movilidad en el Randstad holandés., Madrid., Mo Fomento.

Vence, X y Outes, X.L. (1998). La Unión Europea y la crisis del Estado del Bienestar., Madrid., Edt. Síntesis., 429 págs.

Vázquez Barquero., A. (1993). Política económica local., Madrid., Edt. Pirámide., 333 págs.

VERCHER, A., (1998), "Derechos Humanos y Medio Ambiente", Claves de Razón Práctica, No 84, Julio-Agosto, Madrid. 
VILLAMIL SERRANO, A., MATÍES GARCÍA, J., (1998), Política Económica del Medio Ambiente, Aplicaciones Empresariales, Colección Ceura, Editorial Centro de Estudios Ramón Areces, Madrid.

VV.AA. (1990): Economía de la pobreza. I.C.E. N.686.

VV.AA. (1991): Desarrollo Humano: informe de 1991. Bogotá. PNUD. Tercer Mundo Editores.

VV.AA. (1992): «Guía de acción joven sobre desarrollo sostenible». Madrid. AIESEC. 280 págs.

Todaro, M.P. (1 989): «Economic Development in the Third World». New York, 41 edc. Longman Inc.

United Nations (2000): Human Development Report 2001. Oxford University Press.

United Nations (2000): Global Outlook 2001. An Economic Social and Environmental Perspective. New York.

Van de Walle, N. (1989): «Privatization in developing countries: a review of the issues». World Development, 1(5). pp. 601-615.

Vazquez Barquero, A. (1998). "Desarrollo local y dinámica regional, las enseñanzas de las experiencias españolas"., en Mella, J.Ma. (coord.). Economía y política regional en España ante la Europa del siglo XXI., Madrid., Edcs. Akal., pp. 60-72.

Vidal, J.M. (1990): Hacia una economía mundial Norte-Sur:frente afrente. Barcelona.Plaza \& Janes-Cambio 16.410 págs.

Vilá Valentí, J. (1980). "El concepto de región"., en AA.VV. La región y la Geografía Española., Valladolid., pp. 13/51.

Vilagrasa, J. (1991). "Conceptos clave y filofofía de la ciencia en geografía"., en Anales de Geografía de la U.C.M., n 11., pp. 49/80.

Vidal, D. (1998). "¿Desarrollo o regresión en el Sur?, Le Monde diplomatique., octubre.

Veltz, P (1999). Mundialización, ciudades y territorios., Barcelona., Adt. Ariel. Woods, R. (1982): Population analysis in geography. London. Longman. 245 págs. 